\title{
A Conquista do MPS.BR Nível F na Qualità Informática: Um Caso de Sucesso
}

\author{
Josiane Brietzke ${ }^{1}$, Pablo A. do Prado López ${ }^{1}$, Isabel Albertuni ${ }^{1}$, Luís A. Richter ${ }^{1}$ \\ ${ }^{1}$ Qualità Informática Ltda \\ Rua 16 de Julho, 42/607 - 90.550-020 - Porto Alegre - RS - Brasil \\ \{josiane.brietzke, pablo.lopez, isabel.albertuni,richter\} @qualita.inf.br
}

\begin{abstract}
The article objective is relate the experience for obtaining the F level in the MPS.BR (Brazilian Software Process Improvement) model in Qualità Informática Company, it strategy for process definition and implantation, founded difficulties, success factors, main results and learned lessons. This experience consists on a typical case for process qualification in small business, Brazilian software company MPS.BR's program focus with Brazilian Software Excellence for Promotion Association (SOFTEX) financial subsidies.

Resumo. O objetivo deste artigo é relatar a experiência de obtenção do Nível $F$ do Modelo de Referência do programa de Melhoria de Processos de Software Brasileiro (MPS.BR) na Qualità Informática, sua estratégia para definição e implantação dos processos, dificuldades encontradas, fatores de sucesso, principais resultados obtidos e lições aprendidas. Esta experiência consiste num caso típico de qualificação dos processos de uma pequena organização, pertencente ao grupo de empresas de software brasileira foco do programa MPS.BR, que contou com apoio financeiro da Associação para Promoção da Excelência do Software Brasileiro (SOFTEX).
\end{abstract}

\section{Introdução}

Alcançar competitividade pela qualidade, para as empresas de software, implica tanto na melhoria da qualidade dos produtos de software e serviços correlatos, como dos processos de produção e distribuição de software [MPS.BR 2006]. Diante disto e de objetivos estratégicos, em setembro de 2005, a Qualità Informática aderiu ao projeto Cooperativa MPS.BR - SOFTSUL [MPS.BR 2007a], a fim de implementar o nível Gerenciado (F) do Modelo de Referência (MR-MPS) num período de 15 meses. Este projeto foi coordenado pela Instituição Implementadora (II) Sociedade SulRiograndense de Apoio ao Desenvolvimento de Software (SOFTSUL) e previa a implementação dos níveis de maturidade Parcialmente Gerenciado (G) e Gerenciado (F) em cinco empresas gaúchas.

Logo após a adesão, a Qualità iniciou o planejamento de seu projeto de melhoria de processos para atingimento do MPS.BR Nível $F$ denominado de $\mathrm{Q}^{2}$, definido com o apoio da consultoria e baseado nos resultados obtidos num diagnóstico inicial realizado pela II [Prikladnicki 2005], conquistando este nível de maturidade em janeiro de 2007.

Este artigo está organizado da seguinte forma: a seção 2 apresenta um histórico e características da Qualità; a seção 3 descreve o projeto $Q^{2}$ e sua estratégia para definição 
e implementação dos processos do MPS.BR Nível F; a seção 4 apresenta os resultados obtidos no projeto $\mathrm{Q}^{2}$ e numa pesquisa conduzida internamente; a seção 5 descreve lições aprendidas; e por fim, a seção 6 trata das considerações finais.

\section{A Empresa}

A Qualità Informática iniciou suas atividades em $1^{\circ}$ de agosto de 1991 através do empreendedorismo de três profissionais, que atuavam no núcleo para desenvolvimento de sistemas transacionais com ênfase para automação bancária da Hewleet Packard (antiga EDISA).

Atualmente, possui quatro unidades de negócio: Alocação de Profissionais (UNAP), Varejo (UNVA), Pesquisa \& Desenvolvimento (UNPD) e Fábrica de Software (UNFS). Também conta com cinquienta colaboradores aproximadamente e mais de noventa clientes ativos em estrutura própria, concentrando sua estratégia de atuação no mercado corporativo, focada em serviços especializados (UNAP), soluções de Transferência Eletrônica de Fundos e sistemas de cartões próprios (UNVA), projetos estratégicos e de pesquisa (UNPD) e desenvolvimento de sistemas sob demanda (UNFS), prevendo todo o ciclo de vida, com metodologia própria aderente aos processos do Nível F do MR-MPS [MPS.BR 2006].

A conquista do MPS.BR Nível F pela Qualità foi motivada pelos seguintes objetivos estratégicos: melhorar a qualidade dos produtos e serviços de software; melhorar a satisfação dos usuários, clientes, parceiros e colaboradores; aumentar a participação no mercado nacional; e possibilitar a exportação de software.

\section{O Projeto $Q^{2}$}

O projeto $\mathrm{Q}^{2}$ teve como objetivo principal a definição, implementação e aprimoramento de práticas de engenharia de software das áreas de processo do Nível F do MR-MPS na UNFS cuja equipe era composta de 12 colaboradores.

Este projeto contou com a seguinte estrutura: Comitê Gestor de Qualidade (CGQ) composto pelo Conselho Administrativo (CA) e pela Coordenadora do Grupo de Melhoria de Processo (GMP) e Equipes Técnicas (EQT). O GMP era composto por dois recursos full time e quatro part time. Já as EQTs eram compostas por especialistas da área de processo e pelo menos um participante do GMP e do CA, para manter o alinhamento com os objetivos estratégicos da empresa e acompanhamento das atividades.

O planejamento do projeto teve como base os fatores de resistência identificados em Brietzke (2005) e a estratégia de implementação adotada foi seqüencial, prevendo pacotes de trabalho para cada área de processo: Gerência de Requisitos (GRE), Gerência de Projetos (GPR), Garantia da Qualidade (GQA), Gerência de Configuração (GCO), Medição (MED) e Aquisição (AQU).

O ciclo de melhoria adotado para cada área de processo foi o seguinte:

- Planejamento: definição do pacote de trabalho a ser desenvolvido, alocação da EQT, definição dos participantes em treinamento externo e workshops promovidos pela II, mentoring com a consultoria e distribuição das tarefas; 
- Desenvolvimento: levantamento inicial da documentação e práticas já utilizadas, elaboração de artefatos como fluxos de atividades, políticas, guias, padrões, procedimentos e templates a partir de estudo e pesquisa pela EQT de referências e ferramentas de apoio existentes no mercado. Ao final, a mesma era revisada e aprovada pela EQT e uma baseline do Qualità Framework $(\mathrm{QF})^{1}$ era distribuída;

- Treinamento Interno: capacitação interna realizada pelo GMP para os recursos da UNFS e outros envolvidos no processo, antes da implantação das práticas da área de processo nos projetos piloto;

- Implantação: início da implantação nos projetos piloto a fim de institucionalizar, identificar e avaliar melhorias no processo definido;

- Identificação de Melhorias: tratamento pelo GMP das dificuldades e melhorias encontradas classificando-as em falhas, melhorias ou problemas de institucionalização do processo;

- Acompanhamento dos Projetos Piloto: atualização e aplicação do checklist da auditoria de qualidade nos projetos piloto, a fim de avaliar a aderência, problemas e melhorias identificadas.

Para dar visibilidade do andamento do projeto a toda empresa foram realizadas ações de endomarketing e integrações:

- Kit Boas-Vindas: treinamento de introdução sobre o projeto $\mathrm{Q}^{2}$ para novos colaboradores, abordando os objetivos estratégicos referentes ao projeto $\mathrm{Q}^{2}$, plano de melhoria, fornecendo uma visão geral das atividades e do MR-MPS. Também era entregue aos participantes um bottom com o logo do projeto $\mathrm{Q}^{2}$;

- Boletim Eletrônico $\mathrm{Q}^{2}$ : distribuído mensalmente com informações referentes ao período, atividades em andamento e previstas para o próximo período;

- Momento $\mathrm{Q}^{2}$ : realizado semanalmente para disseminação de conceitos, práticas, conhecimentos e esclarecimento de dúvidas dos processos;

- Concurso $\mathrm{Q}^{2}$ : incentivo à leitura do $\mathrm{QF}$ para disseminação das práticas e criação de banco de questões para exercícios;

- Avaliação a Vista: apoio emocional para os envolvidos na Avaliação Formal através de dinâmicas e reflexões em grupo.

As integrações eram planejadas no atingimento de resultados parciais como, por exemplo, Kick-off do Projeto $\mathrm{Q}^{2}$, logo após a Avaliação Preliminar das Metas Físicas 1 e 2 e Avaliação Informal e Formal descritas na seção 4.2.

Os principais riscos e dificuldades encontradas foram: indisponibilidade de tempo da equipe da organização, priorização sucessiva de outros projetos em função do projeto $\mathrm{Q}^{2}$, resistência à mudança, dificuldade para identificação dos quatro projetos piloto obrigatórios, falta de disseminação da cultura de qualidade e processos na empresa.

\footnotetext{
1 Definição dos processos da Qualità disponibilizada em versão html para consulta de todos os colaboradores e sob responsabilidade do GMP.
} 


\section{Resultados Obtidos}

Nesta seção são apresentados os resultados obtidos numa pesquisa sobre o projeto $Q^{2}$, bem como os resultados alcançados neste projeto, sendo alguns destes identificados de forma similar por outras organizações em Ferreira (2005) e Azevedo (2004).

\subsection{Pesquisa sobre o Projeto $Q^{2}$}

A aplicação desta pesquisa foi realizada de forma confidencial e logo após a conquista do nível de maturidade, durante o mês de fevereiro de 2007. O objetivo foi obter a opinião e a percepção dos envolvidos sobre o projeto a fim de avaliarmos esta experiência e utilizá-la para o planejamento de futuros projetos de melhoria de processos na empresa.

A primeira parte da pesquisa contemplava questões relacionadas aos participantes do projeto a fim de obter o perfil dos mesmos. Na segunda parte, havia questões relacionadas ao projeto, como nível de contribuição das ações planejadas e realizadas para a conquista do nível de maturidade e benefícios obtidos com o projeto.

A seguir, é apresentado o perfil dos envolvidos no projeto $\mathrm{Q}^{2}$. Observa-se, na Figura 1 abaixo, que a maioria dos participantes possui até 5 anos de atuação na empresa, condizente com o tempo de existência da UNFS (escopo da avaliação) e investimentos realizados para sua estruturação pela contratação de novos profissionais. Na Figura 2, nota-se que embora o projeto tenha tido duração de 16 meses na Qualità, a maioria dos recursos humanos alocados para o projeto foram mantidos e isto caracteriza o apoio efetivo da alta direção no patrocínio e proteção destes recursos.

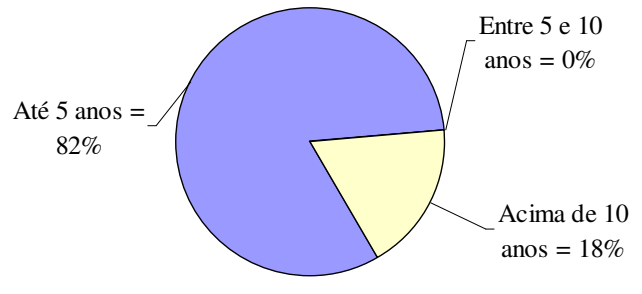

Figura 1. Tempo de atuação dos participantes na Qualità

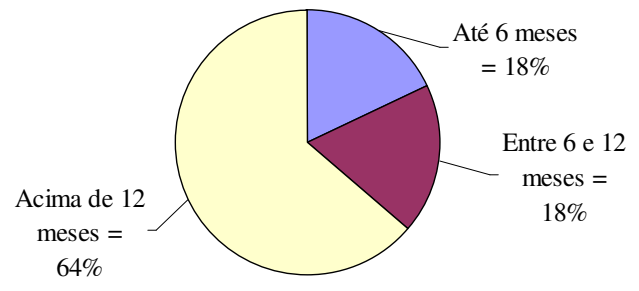

Figura 2. Tempo de atuação dos participantes no projeto $Q^{2}$

Em relação à formação acadêmica, observa-se na Figura 3 que os participantes caracterizam-se como um grupo multidisciplinar, predominando profissionais de Tecnologia da Informação, mas incluindo outras áreas (Matemática e Psicologia).

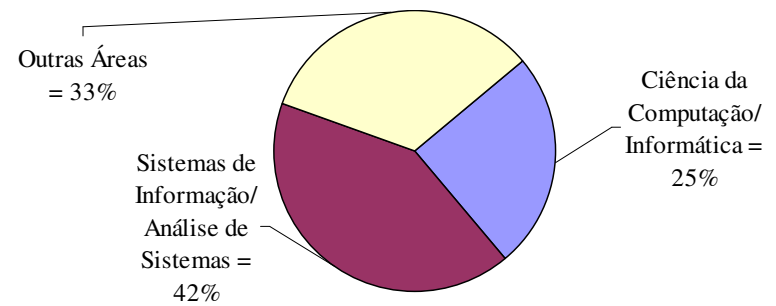

Figura 3. Formação acadêmica dos participantes

Percebe-se na Figura 4 abaixo, que os participantes possuíam experiência intermediária na área de qualidade e melhoria de processos por contar com um implementador MPS.BR credenciado ao SOFTEX e envolvidos em iniciativa anterior 
da empresa baseada no modelo SW-CMM [PAULK 1993]. Por outro lado, a maioria dos participantes $(60 \%)$ possuíam nenhuma (10\%) e baixa experiência anterior (50\%).

Quando questionados sobre a contribuição do projeto $\mathrm{Q}^{2}$ para a experiência profissional dos participantes, observa-se na Figura 5 que a maioria considera de alto valor. Percebe-se com este resultado uma forte motivação à participação em futuras iniciativas de qualidade e melhoria de processos, que a empresa venha a estabelecer.

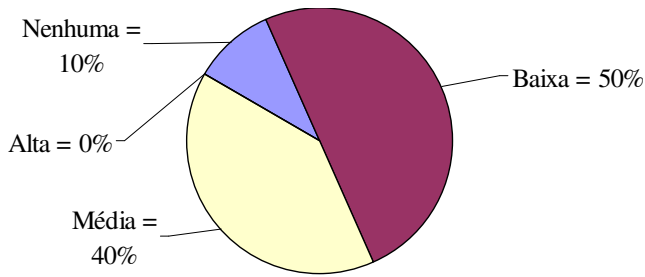

Figura 4. Experiência anterior em qualidade e melhoria de processos

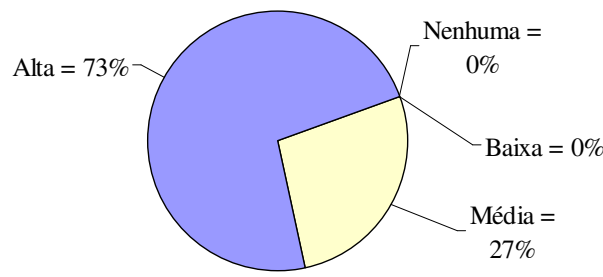

Figura 5. Contribuição para a experiência profissional

Conforme pode ser visto na seção 3, diversas ações foram planejadas durante o projeto $\mathrm{Q}^{2}$ com a finalidade de minimizar seus riscos relacionados ao fator humano, cultura e institucionalização dos processos. Na opinião dos participantes, as ações que mais contribuíram para obtenção do MPS.BR Nível F pela Qualità foram as edições do Momento $\mathrm{Q}^{2}$, o Ciclo de Melhoria adotado e os Treinamentos Internos. Em segundo lugar, ações referentes à alocação e estrutura do CGQ, mentoring com a equipe de qualidade, QF e reuniões de análise e solução de problemas nos projetos e processos.

Já as ações menos votadas pelos participantes, serão reavaliadas para uma futura iniciativa de qualidade e melhoria de processos: Avaliação (prova) sobre área de processo, Avaliação a Vista, Boletim Eletrônico $Q^{2}$ e o Concurso $Q^{2}$.

Em relação aos benefícios obtidos com o Projeto $Q^{2}$, os participantes foram unânimes e consideraram que os principais foram padronização, melhoria de processos, existência de uma avaliação mais objetiva com resultados de auditorias e indicadores, aprendizado, controle e canais de comunicação bem definidos.

\subsection{Resultados do Projeto $Q^{2}$}

$\mathrm{O}$ projeto $\mathrm{Q}^{2}$ teve o estabelecimento de algumas metas, denominadas Metas Físicas (MFs) que deveriam ser atingidas, para que o cronograma do projeto cooperado fosse cumprido, viabilizando o apoio financeiro fornecido pelo SOFTEX [MPS.BR 2007b]. As Avaliações Preliminares estavam relacionadas às MFs, que previam a liberação de determinado percentual de recursos se fossem atingidas.

Os resultados das MFs foram obtidos através de avaliação on site pela II registrada numa planilha, onde caracterizavam os graus de definição e implantação da empresa em cada área de processo. Por exemplo, para o resultado de $100 \%$ de Implantação de GRE, a Qualità obteve grau Totalmente Implementado para todos os resultados esperados e atributos de processo de GRE - Nível F na Avaliação Preliminar da MF2, conforme resultados apresentados na Tabela 1 abaixo. 
Tabela 1. Metas Físicas e Resultados da Qualità

\begin{tabular}{|c|c|c|c|c|}
\hline $\begin{array}{l}\text { Meta } \\
\text { Física } \\
(\text { MF) }\end{array}$ & Prazo & $\begin{array}{c}(\%) \\
\text { Recursos } \\
\text { Liberados }\end{array}$ & Resultado Esperado & Resultado Qualità \\
\hline MFO & Inicial & $30 \%$ & $\begin{array}{l}\text { Adesão das } \mathbf{5} \text { empresas ao } \\
\text { projeto Cooperativa MPS.BR - } \\
\text { SOFTSUL. }\end{array}$ & $\begin{array}{l}\text { Adesão ao projeto Cooperativa MPS. } \\
\text { BR em setembro de } 2005 \text {. }\end{array}$ \\
\hline MF1 & $\begin{array}{c}\mathbf{6} \\
\text { meses }\end{array}$ & $30 \%$ & $\begin{array}{l}\text { - Estágio de implementação do } \\
\text { projeto em, no mínimo, } \mathbf{5 0 \%} \\
\text { do } \\
\text { - Ter iniciado } 2 \text { projetos que } \\
\text { deverão ser avaliados. }\end{array}$ & $\begin{array}{l}\text { - 100\% de Definição de GRE e GPR; } \\
\text { - } \mathbf{8 9 \%} \text { de Implantação de GRE e } \mathbf{9 8 \%} \\
\text { de GPR, em } \mathbf{2} \text { projetos piloto. }\end{array}$ \\
\hline MF2 & $\begin{array}{c}12 \\
\text { meses }\end{array}$ & $30 \%$ & $\begin{array}{l}\text { - Estágio de implementação do } \\
\text { projeto em 100\% do planejado; } \\
\text { - Conclusão de } 2 \text { projetos a } \\
\text { serem avaliados; } \\
\text { - Ter iniciado } 2 \text { outros projetos } \\
\text { a serem avaliados; } \\
\text { - Entrega do Plano da } \\
\text { Avaliação Formal prevista para } \\
\text { os próximos } 3 \text { meses. }\end{array}$ & $\begin{array}{l}\text { - } \mathbf{1 0 0 \%} \text { de Definição de GRE, GPR, } \\
\text { GQA, GCO, MED e AQU; } \\
\text { - } \mathbf{1 0 0 \%} \text { de Implantação de GRE, } \\
\text { GPR, GQA, GCO; } \\
\text { - } \mathbf{8 8 \%} \text { de Implantação de MED; } \\
\text { - } \mathbf{2 9 \%} \text { de Implantação de AQU, } \\
\text { sendo considerada não aplicável para } \\
\text { os projetos piloto; } \\
\text { - Entrega de Plano da Avaliação } \\
\text { Formal. }\end{array}$ \\
\hline MF3 & $\begin{array}{c}15 \\
\text { meses }\end{array}$ & $10 \%$ & $\begin{array}{l}\text { Realização da Avaliação } \\
\text { Formal e aprovação da } \\
\text { "organização". }\end{array}$ & Obtenção do Nível F. \\
\hline
\end{tabular}

A Avaliação Informal (AI) foi conduzida por consultores da II e foram atingidos os seguintes resultados por área de processo do Nível F:

Tabela 2. Resultados da Avaliação Informal

\begin{tabular}{|l|c|c|c|c|c|c|}
\hline \multicolumn{1}{|c|}{ Resultado } & GRE & GPR & GCO & GQA & MED & AQU \\
\hline Implementado & & $\mathrm{X}$ & & $\mathrm{X}$ & & \\
\hline Largamente Implementado & $\mathrm{X}$ & & $\mathrm{X}$ & & & \\
\hline Parcialmente Implementado & & & & & $\mathrm{X}$ & \\
\hline Não Aplicável & & & & & & $\mathrm{X}$ \\
\hline
\end{tabular}

Conforme caracterização de processos da AI, o resultado Implementado é atribuído ao processo que gera todos os resultados esperados e atende todos os atributos de processo previstos no MR-MPS, sem pontos fracos. Largamente Implementado significa que o processo gera a maioria dos resultados esperados e atende a maioria dos atributos de processo, enquanto que Parcialmente Implementado, é atribuído ao processo que gera alguns dos resultados esperados, podendo também atender a algum (ou alguns) dos atributos de processo. Não Aplicável significa que o processo não se aplica ao âmbito da organização.

Após a AI, que teve como objetivo principal identificar desvios em relação ao MR-MPS, o GMP elaborou um plano de ação para tratamento dos mesmos até a Avaliação Formal (AF) e conseqüentemente, atingimento da MF3. A AF foi conduzida pela Instituição Avaliadora (IA) SWQuality no período de 22 a 24 de janeiro de 2007 e o resultado foi Satisfeito para o MPS.BR Nível F [MPS.BR 2007c]. 


\section{Lições Aprendidas}

As lições aprendidas foram segmentadas em pontos fortes, fracos e lições genéricas, sendo as mais relevantes citadas abaixo.

Pontos Fortes:

- Apoio efetivo da alta direção;

- Comprometimento, união e empenho de todos os envolvidos para atingir as metas do projeto;

- Experiência e apoio dos consultores, atendimento remoto e conhecimento prático para definição de processos e esclarecimentos de dúvidas;

- Atuação da área de qualidade, auditores e auditados em quesitos como,próatividade, postura e criatividade;

- Alocação de dois recursos full time na área da qualidade;

- Gestão de riscos do projeto, pois garantiu a tomada de decisões e o contorno de dificuldades dentro do prazo necessário para não prejudicar o andamento do projeto.

Pontos Fracos:

- Limitação de recursos envolvidos (sobreposição de papéis, superalocação de recursos, infra-estrutura e orçamento) por ser uma organização pequena;

- Centralização das mudanças do processo na área de Qualidade, que além de atender um pacote novo em desenvolvimento, gerenciava as melhorias identificadas na aplicação dos processos nos projetos;

- Inexistência de um canal mais ágil para comunicação (intranet) para publicação do QF, Boletim Eletrônico do $\mathrm{Q}^{2}$ e notícias em geral;

- Controle parcial do esforço e do custo do projeto $\mathrm{Q}^{2}$.

Lições Genéricas:

- Falta de piloto do QF com cliente externo, pois os projetos escolhidos para avaliação correspondem a produtos internos;

- Identificação de todas as dependências críticas com antecedência.

\section{Considerações Finais}

A conquista do MPS.BR Nível F permite à Qualità o planejamento de novas ações voltadas à área de qualidade e, desta forma, a consolidação de seus processos, tanto no contexto de desenvolvimento de software, como no contexto organizacional, para possibilitar o amadurecimento profissional de seus colaboradores, tornando a organização mais competitiva no mercado.

A disseminação da cultura de qualidade e de processos para as outras unidades de negócio e áreas de apoio da empresa está sendo implantada, possibilitando que a empresa unifique seu padrão de gestão. Adicionalmente, estão sendo estudadas ações de marketing para divulgação e consolidação da imagem da empresa. 
Dentre as iniciativas previstas a curto prazo, está a adesão ao Programa Gaúcho de Qualidade e Produtividade (PGQP), cujos resultados de diagnóstico deste programa mostraram-se alinhados aos objetivos estratégicos e compatíveis com parte das práticas do MR-MPS já implementadas na organização como, por exemplo, as práticas do processo de MED.

Para médio prazo, planeja-se a adesão em níveis superiores de maturidade do MPS.BR, bem como busca de avaliação Capability Maturity Model Integration (CMMi) em nível equivalente ou superior, a fim de ampliar o mercado de atuação da empresa, visando exportação de software.

$\mathrm{O}$ projeto $\mathrm{Q}^{2}$ obteve diversas conquistas, além da própria certificação da empresa e, ao longo deste processo, percebeu-se um crescente e valioso espírito de equipe, onde cada um empenhou-se em cumprir seu papel para o atingimento do objetivo comum de todos. O êxito do projeto foi confirmado não somente na AF, mas também pelos resultados obtidos e pela grande diferença entre o número de pontos fortes (23) e fracos (5) apontados pela equipe, decisivos para o sucesso do projeto $\mathrm{Q}^{2}$.

\section{Referências}

Azevedo, Raimundo S. N. et al. (2004) “Certificação ISO 9001:2000 - a Experiência da SoftExport”, III SBQS, Brasília, Distrito Federal.

Brietzke, Josiane; Rabelo, Abraham (2005) "Estudo sobre Fatores que Influenciam em Projetos de Melhoria de Processos de Software", In: Jornadas Chilenas de Computación, Universidad Austral de Chile, Chile.

Ferreira, Analia I. F. et al. (2005) "Implantação de Processo de Software na BL Informática - Um Caso de Sucesso", IV SBQS, Porto Alegre, Rio Grande do Sul.

MPS.BR (2006) "SOFTEX - Associação para Promoção da Excelência do Software Brasileiro. MPS.BR - Guia Geral (Versão 1.1)", http://www.softex.br/mpsbr/_guias/MPS.BR_Guia_Geral_V1.1.pdf.

MPS.BR (2007a) "SOFTEX - Associação para Promoção da Excelência do Software Brasileiro. MPS.BR - Implementações em Grupos de Empresas- Grupo de Empresas G1”, http://www.softex.br/mpsbr/_implementacoes/MPS.BR_SOFTSUL.pdf.

MPS.BR (2007b) "SOFTEX - Associação para Promoção da Excelência do Software Brasileiro. MPS.BR, Implementações em Grupos de Empresas, Planilha Financeira,, G1”,http://www.softex.br/mpsbr/_implementacoes/MPS.BR_PFIN_SOFTSUL.pdf.

MPS.BR (2007c) "SOFTEX - Associação para Promoção da Excelência do Software Brasileiro. MPS.BR - Avaliações MA-MPS - Qualità - Nível F do MPS.BR”, http://www.softex.br/mpsbr/_avaliacoes/avaliacao.asp?id=1434.

PAULK, Mark C. et al. (1993) "Capability Maturity Model for Software, Version 1.1", http://www.sei.cmu.edu/pub/documents/93.reports/pdf/tr24.93.pdf.

Prikladnicki, Rafael et al. (2005) "Uma Abordagem para a Realização de Diagnóstico Inicial em Empresas que Implementam o MPS.BR", In: I Encontro de Implementadores do MPS.BR, Universidade Católica de Brasília, Brasil. 